\title{
A fractional Malthusian growth model with variable order using an optimization approach
}

\author{
Ricardo Almeida $^{1, *}$, Nuno R. O. Bastos ${ }^{1,2}$, M. Teresa T. Monteiro ${ }^{3}$ \\ ${ }^{1}$ Center for Research and Development in Mathematics and Applications (CIDMA), \\ Department of Mathematics, University of Aveiro, 3810-193 Aveiro, Portugal \\ ${ }^{2}$ Department of Mathematics, School of Technology and Management of Viseu, Polytechnic Institute of Viseu, 3504-510 Viseu, Portugal \\ ${ }^{3}$ Algoritmi R\&D Center, Department of Production and Systems, University of Minho, Campus de Gualtar, 4710-057 Braga, Portugal
}

\begin{abstract}
The objective is to study the population's growth with a fractional differential equation. The order of the fractional derivative is a function depending on time and the goal is to determine the fractional order function that better fits the given data. The model is than tested to describe the world population growth and of some countries. All the numerical experiments were done in MATLAB, using the routines lsqcurvefit, fminunc and spline.
\end{abstract}

Keywords Fractional calculus, fractional differential equation, least squares

AMS 2010 subject classifications 26A33, 34A08, 90C30

DOI: $10.19139 /$ soic.v6i1.465

\section{Introduction}

The Malthusian growth model was proposed in 1798 by the English economist Thomas Malthus in his book $A n$ Essay on the Principle of Population. The theory states that population number has exponential growth based on a constant rate, applied to ideal circumstances or to a short period of time, when an individual lives in a region with no constraints on food and with no natural enemies. In this case, if $N(t)$ represents the size of the population at an instant $t$, the dynamic differential equation

$$
N^{\prime}(t)=P \cdot N(t)
$$

models the growth of the population. The constant $P$, called the Malthusian parameter, is given by the difference between the fertility and the mortality rates, assuming that these rates are constant in time. If $N_{0}$ is the initial level of the population, the function

$$
N(t)=N_{0} \exp (P t)
$$

gives the exact number of individuals at a given time $t$. In reality, the growth of a population has some limitations due to environments restrictions, like food availability, competition with other species, competition for territory, etc. To model more realistic population growth, the Belgian mathematician Pierre Verhulst in 1838 proposed another formula, that illustrates how a population may increase exponentially until it reaches the carrying capacity of its environment. This model is known as the Logistic equation and it is given by the differential equation

$$
N^{\prime}(t)=P \cdot N(t)\left(1-\frac{N(t)}{K}\right)
$$
*Correspondence to: Ricardo Almeida (Email: ricardo.almeida@ua.pt). Department of Mathematics, University of Aveiro, 3810-193
Aveiro, Portugal

ISSN 2310-5070 (online) ISSN 2311-004X (print)

Copyright (C) 2018 International Academic Press 
where $P$ is the Malthusian parameter (the rate of population growth) and $K$ is the carrying capacity, that is, the maximum sustainable population. The solution to this problem is given by the curve

$$
N(t)=\frac{K}{1+\left(K / N_{0}-1\right) \exp (-P t)} .
$$

Although there are several models to describe the dynamics of the population growth, the Malthusian model has the advantage that it is given by a linear differential equation. Later, when we model the same problem but using a fractional differential equation, we know the analytic expression of the solution for the problem.

To test the different models that we propose here, we will see how close they are to real data, by fitting the solution with dependence on some parameters with the observations. One of the most used methods is the least squares technique. Suppose that the data consists in $m$ points, say $\left(t_{0}, x_{0}\right), \ldots,\left(t_{m}, x_{m}\right)$, and we intend to fit these values in a theoretical model $t \mapsto x(t)$, where the form $x$ is known but it depends on some unknown parameters $\beta_{1}, \ldots, \beta_{k}$. If we consider in each step the error $d_{i}:=x_{i}-x\left(t_{i}\right)$, for $i=0, \ldots, m$, then the total error is given by

$$
E:=\sum_{i=0}^{m}\left(d_{i}\right)^{2} .
$$

The goal is to find the values of the parameters $\beta_{1}, \ldots, \beta_{k}$ for which $E$ attains a minimum value.

For the readers convenience, we present here a short introduction on the fractional calculus theory. Following $[10,16]$, fractional derivatives are a generalization of ordinary derivatives, by considering an arbitrary real positive order. This subject has gain great importance during the past decades, since we can describe the dynamics of certain phenomena better when considering the problem modeled by a fractional differential equation. For example, we find applications in physics [7, 8], chemistry [11, 28], computer science [9, 15], engineering [18, 23, 24, 26], viscoelastic $[2,13]$, systems theory $[3,12]$ etc.

Two of the most important concepts in this theory are due to Riemann, and are the following. If $x$ is a real valued integrable function in $[a, b]$ and $\alpha$ a positive real, the Riemann-Liouville fractional integral of $x$ of order $\alpha$ is given by the integral

$$
I_{a+}^{\alpha} x(t):=\frac{1}{\Gamma(\alpha)} \int_{a}^{t}(t-\tau)^{\alpha-1} x(\tau) d \tau
$$

When $\alpha$ is an integer, this expression is an n-tuple integral of form

$$
\int_{a}^{t} d \tau_{1} \int_{a}^{\tau_{1}} d \tau_{2} \ldots \int_{a}^{\tau_{n-1}} x\left(\tau_{n}\right) d \tau_{n}
$$

For the fractional derivative, let $n \in \mathbb{N}$ be such that $\alpha \in(n-1, n)$. The Riemann-Liouville fractional derivative of $x$ of order $\alpha$ is defined as

$$
D_{a+}^{\alpha} x(t):=\frac{1}{\Gamma(n-\alpha)}\left(\frac{d}{d t}\right)^{n} \int_{a}^{t}(t-\tau)^{n-\alpha-1} x(\tau) d \tau .
$$

We remark that, in opposite to ordinary derivatives, these fractional operators are nonlocal and contain memory and thus may be suitable when the current moment is most influenced by the past process. More recently, a similar concept was given, due to Michele Caputo [4]. If $x \in C^{n}[a, b]$ and given $\alpha \in(n-1, n)$, the Caputo fractional derivative of $x$ of order $\alpha$ is defined as

$$
{ }^{C} D_{a+}^{\alpha} x(t):=\frac{1}{\Gamma(n-\alpha)} \int_{a}^{t}(t-\tau)^{n-\alpha-1} \frac{d^{n} x}{d \tau^{n}}(\tau) d \tau .
$$

Two important results are the relations between the fractional integral and the Caputo fractional derivative, to know

$$
I_{a+}^{\alpha}{ }^{C} D_{a+}^{\alpha} x(t)=x(t)-\sum_{k=0}^{n-1} \frac{x^{(k)}(a)}{k !}(t-a)^{k},
$$


and

$$
{ }^{C} D_{a+}^{\alpha} I_{a+}^{\alpha} x(t)=x(t)
$$

A natural generalization of the previous concepts is to consider the order of the fractional derivative $\alpha$ to be a function $\alpha(\cdot)$, and so it may vary trough time. As it is known, fractional derivatives are nonlocal operators and they contain memory, and so it is reasonable to consider that the order of the derivative is not constant, and depends on time also. This subject is very recent, and was suggested by the first time by Samko and Ross [17]. If $\alpha:[a, b] \rightarrow(n-1, n)$ is the fractional variable order, fractional integrals and fractional derivatives are defined as

$$
I_{a+}^{\alpha(t)} x(t):=\frac{1}{\Gamma(\alpha(t))} \int_{a}^{t}(t-\tau)^{\alpha(t)-1} x(\tau) d \tau
$$

and

$$
D_{a+}^{\alpha(t)} x(t):=\frac{1}{\Gamma(n-\alpha(t))}\left(\frac{d}{d t}\right)^{n} \int_{a}^{t}(t-\tau)^{n-\alpha(t)-1} x(\tau) d \tau,
$$

respectively. In a similar way, a Caputo fractional derivative of variable order can be defined as (see e.g. [20])

$$
{ }^{C} D_{a+}^{\alpha(t)} x(t):=\frac{1}{\Gamma(n-\alpha(t))} \int_{a}^{t}(t-\tau)^{n-\alpha(t)-1} \frac{d^{n} x}{d \tau^{n}}(\tau) d \tau .
$$

In particular, when $\alpha(\cdot)$ takes values in the open interval $(0,1)$, this expression is written as

$$
{ }^{C} D_{a+}^{\alpha(t)} x(t)=\frac{1}{\Gamma(1-\alpha(t))} \int_{a}^{t}(t-\tau)^{\alpha(t)} x^{\prime}(\tau) d \tau .
$$

Theorem 1

If $x(t)=(t-a)^{\gamma}$ with $\gamma>n-1$, we have that when $\alpha([a, b]) \subseteq(n-1, n)$,

$$
{ }^{C} D_{a+}^{\alpha(t)} x(t)=\frac{\Gamma(\gamma+1)}{\Gamma(\gamma-\alpha(t)+1)}(t-a)^{\gamma-\alpha(t)} .
$$

Proof

First, observe that

Using the definition, we obtain

$$
x^{(n)}(t)=\frac{\Gamma(\gamma+1)}{\Gamma(\gamma+1-n)}(t-a)^{\gamma-n} .
$$

$$
\begin{aligned}
{ }^{C} D_{a+}^{\alpha(t)} x(t) & =\frac{\Gamma(\gamma+1)}{\Gamma(\gamma+1-n) \Gamma(n-\alpha(t))} \int_{a}^{t}(t-\tau)^{n-\alpha(t)-1}(\tau-a)^{\gamma-n} d \tau \\
& =\frac{\Gamma(\gamma+1)}{\Gamma(\gamma+1-n) \Gamma(n-\alpha(t))}(t-a)^{n-\alpha(t)-1} \int_{a}^{t}\left(1-\frac{\tau-a}{t-a}\right)^{n-\alpha(t)-1}(\tau-a)^{\gamma-n} d \tau .
\end{aligned}
$$

With the change of variables $s=(\tau-a) /(t-a)$, and recalling the Beta function

$$
B(x, y):=\int_{0}^{1} s^{x-1}(1-s)^{y-1} d s, \quad x, y>0
$$

we deduce the following:

$$
\begin{aligned}
{ }^{C} D_{a+}^{\alpha(t)} x(t) & =\frac{\Gamma(\gamma+1)}{\Gamma(\gamma+1-n) \Gamma(n-\alpha(t))}(t-a)^{\gamma-\alpha(t)} B(n-\alpha(t), \gamma-n+1) \\
& =\frac{\Gamma(\gamma+1)}{\Gamma(\gamma+1-n) \Gamma(n-\alpha(t))}(t-a)^{\gamma-\alpha(t)} \frac{\Gamma(n-\alpha(t)) \Gamma(\gamma-n+1)}{\Gamma(\gamma-\alpha(t)+1)} \\
& =\frac{\Gamma(\gamma+1)}{\Gamma(\gamma-\alpha(t)+1)}(t-a)^{\gamma-\alpha(t)} .
\end{aligned}
$$

This completes the proof. 
We remark that, when $\gamma=k \in \mathbb{N}$ with $k \leq n-1$, from the definition of fractional derivative, it results that

$$
{ }^{C} D_{a+}^{\alpha(t)}(t-a)^{k}=0 .
$$

In opposite to constant fractional order, this variable fractional order operator has no inverse operation like the Riemann-Liouville integral with respect to the Caputo derivative. For this reason, dealing and solving fractional differential equations for variable order is an extremely difficult problem, and often numerical methods are used $[5,25,27]$.

The outline of this paper is the following. In Section 2 we present the Malthusian growth model. We start with the classical model, whose solution is given by an exponential function, and then we replace the ordinary derivative by a fractional derivative, and by doing so the solution is now given by the Mittag-Leffler function. Next, we substitute the fractional constant order $\alpha$ by a function $\alpha(t)$ and with it we model the problem by a variable fractional order dynamics. The goal is to determine the values of the parameters in each model that better fits with given data, and compare the error for each case. In the following Section 3 we proceed with a similar study, but considering this time four countries (China, India, USA and Indonesia) and we compare the classical model with the variable order fractional model. In the numerical experiments, some routines from Mat lab are used.

\section{World Population Growth}

In [1], a fractional approach was considered to model the World Population Growth. Starting with the classical model

$$
N^{\prime}(t)=P \cdot N(t),
$$

the ordinary derivative was replaced by the Caputo fractional derivative, and the dynamic was described by the fractional differential equation

$$
{ }^{C} D_{0+}^{\alpha} N(t)=P \cdot N(t), \quad t \geq 0, \alpha \in(0,1) .
$$

The solution to the fractional problem is given by the function

$$
N(t)=N_{0} E_{\alpha}\left(P t^{\alpha}\right),
$$

where $E_{\alpha}(\cdot)$ denotes the Mittag-Leffler function:

$$
E_{\alpha}(z):=\sum_{k=0}^{\infty} \frac{z^{k}}{\Gamma(k \alpha+1)}, \quad z \in \mathbb{R} .
$$

We remark that, when $\alpha=1$, the Mittag-Leffler function is simply the exponential function, and so the fractional solution coincides with the classical one

$$
N(t)=N_{0} \exp (P t) .
$$

Then, using the data available from the United Nations [21] from year 1910 until 2010, the best values for the parameters were found. To get a better accuracy for the model, the fractional order $\alpha$ was considered free, without any constraints. For the classical model (1), the values obtained were

$$
P \approx 1.3501 \times 10^{-2} \text { with error } \quad E_{\text {classical }} \approx 7.0795 \times 10^{5} .
$$

When we considered the problem modeled by the fractional differential equation (2), the values were

$$
\alpha \approx 1.3933, \quad P \approx 3.4399 \times 10^{-3} \text { with error } \quad E_{\text {fractional }} \approx 2.0506 \times 10^{5} .
$$

So, from these results, we see that the fractional approach is more efficient in modelling the problem than the ordinary one. These numerical tests were done in Matlab [14] using the routine lsqcurvefit that solves 
nonlinear data-fitting problems in least-squares sense. This routine is based on an iterative method with local convergence, i.e., depending on the initial approximation to the parameters to estimate. In the computational tests the trust-region-reflective algorithm was selected. Theoretical details can be found in [19, 22].

The next step is to consider even a more general approach to this problem, by considering the fractional order to be a function depending on time $t \mapsto \alpha(t)$. Motivated by Eq. (3), and considering the Mittag-Leffler function with variable order

$$
E_{\alpha(t)}(z):=\sum_{k=0}^{\infty} \frac{z^{k}}{\Gamma(k \alpha(t)+1)}, \quad z \in \mathbb{R},
$$

we propose the following theoretical model to study the world population problem:

$$
N(t)=N_{0} E_{\alpha(t)}\left(P t^{\alpha(t)}\right) .
$$

Observe that, when $\alpha(\cdot)$ is constant, $\alpha(t) \equiv \alpha$, then expression (5) reduces to expression (3), which in turn when $\alpha \rightarrow 1$, we obtain the classical model (4). We test model (5) by the closeness to the observed data, from which we infer the values of the parameters. To start, we compare the fractional model with constant order with three new ones, with variable fractional orders. For example, we consider fractional derivatives with orders

$$
\alpha_{1}(t):=a t^{2}+b t+c, \quad \alpha_{2}(t):=\cos (a t+b), \quad \text { and } \quad \alpha_{3}(t):=\exp (a t+b),
$$

for some parameters $a, b, c \in \mathbb{R}$. For these cases, we determine the values of the parameters that better fit with the given data using the same routine lsqcurvefit. In Table 1 we summarize the obtained results, and the respective errors.

\begin{tabular}{cccccc}
\hline$\alpha(t)$ & $a$ & $b$ & $c$ & $P$ & $E_{\text {fractional }}$ \\
\hline$\alpha_{1}(t)$ & $-4.4865 \times 10^{-5}$ & $7.5331 \times 10^{-3}$ & 0.8560 & $7.5849 \times 10^{-3}$ & $1.48133 \times 10^{4}$ \\
\hline$\alpha_{2}(t)$ & $-9.8731 \times 10^{-3}$ & 0.8660 & - & $1.3880 \times 10^{-2}$ & $1.7250 \times 10^{4}$ \\
\hline$\alpha_{3}(t)$ & $-1.2401 \times 10^{-3}$ & 0.9117 & - & $2.1398 \times 10^{-4}$ & $6.3825 \times 10^{4}$ \\
\hline
\end{tabular}

Table 1. World population from 1910 until 2010.

From these values, we observe that we already obtain better results, even when compared to the fractional model with constant order. In these cases, we considered some particular fractional orders, but other orders could be considered in order to improve the method. We remark that we do not impose any restriction on $\alpha\left(t_{i}\right)$ in order to obtain a better accuracy for the procedure. Anyway, when $\alpha(t) \equiv \alpha$, that is, when the fractional order is constant, and as $\alpha \rightarrow 1^{ \pm}$, the solution of the fractional differential equation converges to the solution of the differential equation obtained when $\alpha=1$ ( $\mathrm{cf}[1$, Theorem 1]).

One more interesting question is to determine the function $t \mapsto \alpha(t)$ that better fits with the data. Since we are working with discrete data, we are interested in finding the optimal values for $\alpha\left(t_{i}\right)=\alpha_{i}$, in each instant of time $t_{i}$ and also the $P$ value. A nonlinear unconstrained optimization problem is formulated:

$$
\min _{\alpha_{i}, P} \sum_{i=0}^{m}\left(x_{i}-N_{0} E_{\alpha_{i}}\left(P t_{i}^{\alpha_{i}}\right)\right)^{2}
$$

whose variables are $\alpha_{i}, i=0 \ldots, m$, and $P$.

In our case study, the data $\left(t_{i}, x_{i}\right)$ consists in 12 values, and we need to find the best values for $\alpha\left(t_{i}\right), i=0, \ldots, 11$ and $P$. The results are then

$$
\begin{aligned}
& \alpha\left(t_{0}\right) \approx 1.0000, \quad \alpha\left(t_{1}\right) \approx 0.7585, \quad \alpha\left(t_{2}\right) \approx 0.9430, \quad \alpha\left(t_{3}\right) \approx 0.9785, \\
& \alpha\left(t_{4}\right) \approx 0.9805, \quad \alpha\left(t_{5}\right) \approx 1.0370, \quad \alpha\left(t_{6}\right) \approx 1.0763, \quad \alpha\left(t_{7}\right) \approx 1.0932, \\
& \alpha\left(t_{8}\right) \approx 1.1015, \quad \alpha\left(t_{9}\right) \approx 1.1002, \quad \alpha\left(t_{10}\right) \approx 1.0999, \quad \alpha\left(t_{11}\right) \approx 1.0931,
\end{aligned}
$$

with $P \approx 9.6850 \times 10^{-3}$. The error that comes from this procedure is

$$
E_{\text {fractional }} \approx 0.1196,
$$


and in Figure 1 we have the plot of the results. On left side, we exhibit the graph of the obtained fractional order curve using the routine spline. On the right side we present a comparison between the data, the classical model and the variable fractional model. In these numerical experiments the fminunc routine from the Mat lab Optimization toolbox is used. This routine attempts to find a minimum of a scalar function of several variables, starting at an initial estimate. The interior-point optimization algorithm was selected.

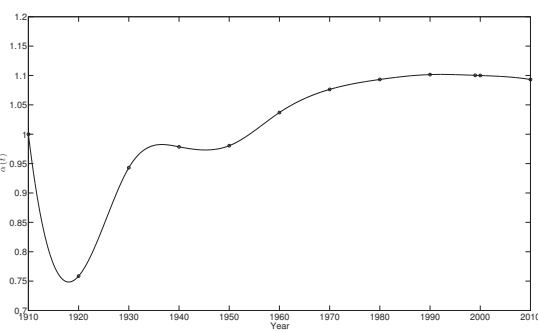

(a) Optimal fractional order

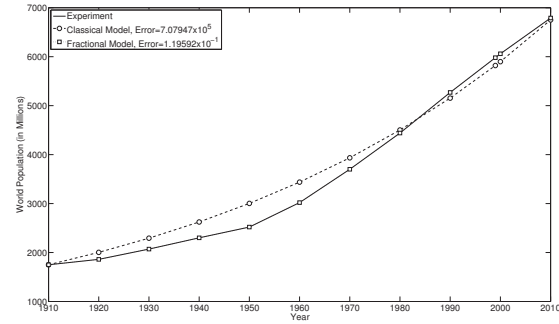

(b) Experimental data vs classical model vs fractional model

Figure 1. World Population Growth.

\section{Countries Population Growth}

In this section we study the population growth model for several countries. We choose the World's four most populous countries in 2015: People's Republic of China, India, USA and Indonesia. For data source, we use the ones available from [6], and we study from the year 1910 until 2010, measured every 10 years, consisting in 11 values. In each case, we determine the best variable fractional order for each country, taking into account the 11 values. In Table 2 we present the growth rates $P$, as well the error, for each country.

\begin{tabular}{ccccc}
\hline Country & $P-$ Classical Model & Error & $P$ - Fractional Model & Error \\
\hline China & $1.1823 \times 10^{-2}$ & $5.0155 \times 10^{4}$ & $9.3059 \times 10^{-3}$ & $8.1067 \times 10^{-4}$ \\
\hline India & $1.5321 \times 10^{-2}$ & $4.2133 \times 10^{4}$ & $9.1063 \times 10^{-3}$ & $3.8354 \times 10^{-3}$ \\
\hline USA & $1.2363 \times 10^{-2}$ & $5.9101 \times 10^{2}$ & $1.3453 \times 10^{-2}$ & $8.8584 \times 10^{-6}$ \\
\hline Indonesia & $1.7417 \times 10^{-2}$ & $8.2862 \times 10^{2}$ & $1.3200 \times 10^{-2}$ & $6.1555 \times 10^{-5}$ \\
\hline
\end{tabular}

Table 2. Population by countries: classical vs fractional models.

In Figure 2 we present the plots of the fractional optimal order for each country, using again the routine spline, and in Figure 3 we compare for each country the data, with the classical and fractional models.

\section{Conclusion}

In this paper we studied the population growth problem, by modeling the dynamics by a fractional differential equation. Improving the results obtained in [1], where the fractional order is constant, here we generalize the previous work by considering the order a function depending on time. As we saw, the variable order model is much more efficient in modelling the World Population Growth, compared to the constant order one. This may suggest that, instead of considering constant order fractional differential equations, in some situations, we can improve the method by considering the order as a function depending on time. This is an important issue, with very few works done in this direction, and we hope to attract the attention of the community to this kind of problems. One 


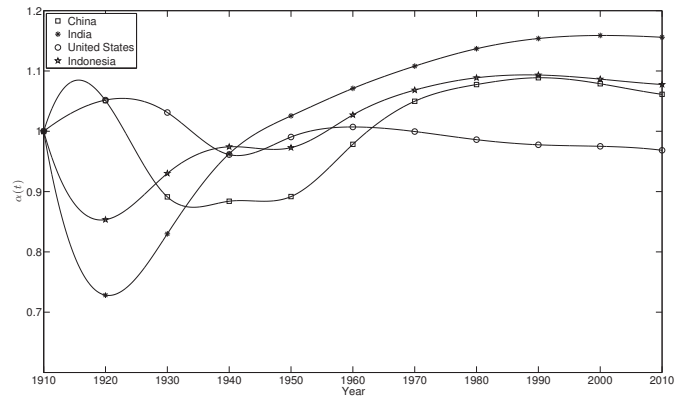

Figure 2. Optimal fractional order.

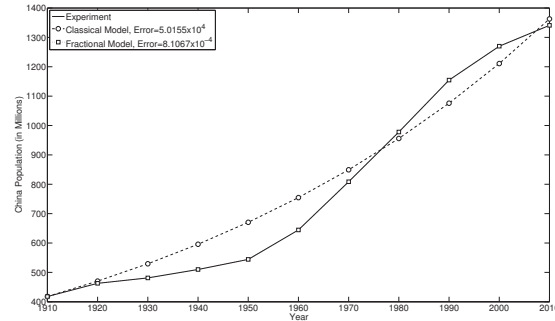

(a) China.

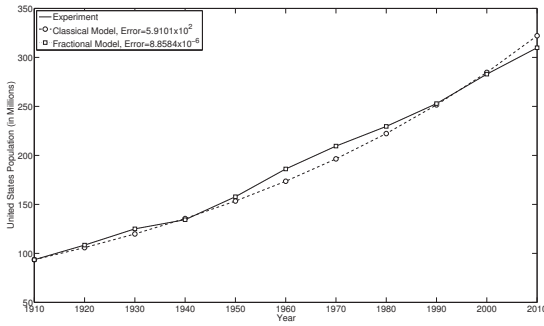

(c) USA.

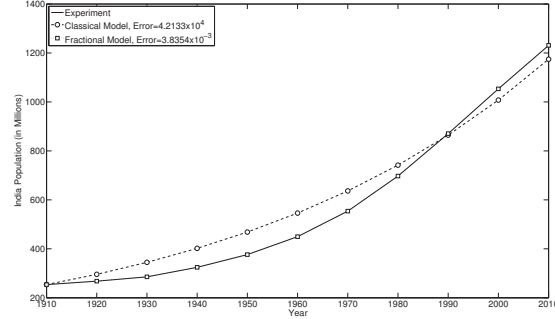

(b) India

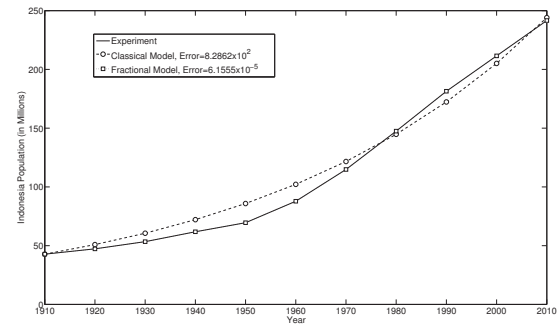

(d) Indonesia

Figure 3. Countries Population Growth.

interesting question, that we hope to answer in the future, is what is the relation between the dynamics of the population growth with the fractional order? Is there a connection between the monotonicity of $\alpha(t)$ and the rate of growth of the population? This will be our next problem to be treated.

\section{Acknowledgements}

The first and second authors were supported by Portuguese funds through the CIDMA - Center for Research and Development in Mathematics and Applications, and the Portuguese Foundation for Science and Technology (FCTFundação para a Ciência e a Tecnologia), within project UID/MAT/04106/2013; third author by the ALGORITMI R\&D Center and project PEst-UID/CEC/00319/2013. 


\section{REFERENCES}

1. R. Almeida, N.R.O. Bastos and M.T.T. Monteiro, Modelling some real phenomena by fractional differential equations, Mathematical Methods in the Applied Sciences, vol. 39, pp. 4846-4855, 2016.

2. R.L. Bagley and P.J. Torvik, On the fractional calculus model of viscoelastic behavior, Journal of Rheology, vol. 30, pp. 133-155, 1986.

3. Y. Cao, Y. Li, W. Ren and Y. Chen, Distributed coordination of networked fractional-order systems, IEEE Transactions on Systems, Man, and Cybernetics, Part B: Cybernetics, vol. 40, pp. 362-370, 2010.

4. M. Caputo, Linear model of dissipation whose $Q$ is almost frequency independent-II, Geophysical Journal of the Royal Astronomical Society, vol. 13, pp. 529-539, 1967.

5. Y. Chen, L. Liu, B. Li and Y. Sun, Numerical solution for the variable order linear cable equation with Bernstein polynomials, Applied Mathematics and Computation, vol. 238, pp. 329-341, 2014.

6. Gapminder World, http://www. gapminder.org/data/

7. J.F. Gómez-Aguilar, M.G. López-López, V.M. Alvarado-Martínez, J. Reyes-Reyes and M. Adam-Medina, Modeling diffusive transport with a fractional derivative without singular kernel, Physica A. Statistical Mechanics and its Applications, vol. 447, pp. 467-481, 2016.

8. R. Herrmann, Folded potentials in cluster physics - a comparison of Yukawa and Coulomb potentials with Riesz fractional integrals, Journal of Physics A: Mathematical and Theoretical, vol. 46, pp. 405203, 2013.

9. H.A. Jalab, R.W. Ibrahim and A. Ahmed, Image denoising algorithm based on the convolution of fractional Tsallis entropy with the Riesz fractional derivative, Neural Computing and Applications, vol. 28, Supplement 1, pp. 217-223, 2017.

10. A.A. Kilbas, H.M. Srivastava and J.J. Trujillo, Theory and Applications of Fractional Differential Equations, North-Holland Mathematics Studies, 204. Elsevier Science B.V., Amsterdam, 2006.

11. Y.-1 Li, H.-q Tang and H.-x. Chen, Fractional-order derivative spectroscopy for resolving simulated overlapped Lorenztian peaks, Chemometrics and Intelligent Laboratory Systems, vol 107, pp. 83-89, 2011.

12. J. Lu, J. Shen, J. Cao and J. Kurths, Consensus of Networked Multi-agent Systems with Delays and Fractional-Order Dynamics, In Consensus and Synchronization in Complex Networks, (Ed. L. Kocarev) 69-110 Springer, 2013.

13. F. Meral, T. Royston and R. Magin, Fractional calculus in viscoelasticity: An experimental study, Communications in Nonlinear Science and Numerical Simulation, vol. 15, pp. 939-945, 2010.

14. C. Moler, J. Little, and S. Bangert, Matlab User's Guide - The Language of Technical Computing, The MathWorks, Sherborn, Mass. (2001).

15. X. Pan, Y. Ye and J. Wang, Fractional directional derivative and identification of blur parameters of motion-blurred image, Signal, Image and Video Processing, vol. 8, pp. 565-576, 2014

16. I. Podlubny, Fractional differential equations, Mathematics in Science and Engineering, 198. Academic Press, Inc., San Diego, CA, 1999.

17. S. G. Samko and B. Ross, Integration and differentiation to a variable fractional order, Integral Transforms and Special Functions, vol. 1, pp. 277-300, 1993

18. M. F. Silva, J. A. T. Machado and A. M. Lopes, Fractional order control of a hexapod robot, Nonlinear Dynamics, vol. 38, pp. 417-433, 2004.

19. T. Steihaug, The Conjugate Gradient Method and Trust Regions in Large Scale Optimization, SIAM Journal on Numerical Analysis, vol. 20, pp. 626-637, 1983.

20. D. Tavares, R. Almeida and D.F.M. Torres, Caputo derivatives of fractional variable order: numerical approximations, Communications in Nonlinear Science and Numerical Simulation, vol. 35, pp. 69-87, 2016.

21. United Nations, The World at Six Billion Off Site, Table 1, World Population From Year 0 to Stabilization, 5, 1999.

22. R.A. Waltz, J. L. Morales, J. Nocedal and D. Orban, An interior algorithm for nonlinear optimization that combines line search and trust region steps, Mathematical Programming, vol. 107, pp. 391-408, 2006.

23. D. Wollscheid and A. Lion, The benefit of fractional derivatives in modelling the dynamics of filler-reinforced rubber under large strains: a comparison with the Maxwell-element approach, Computational Mechanics, vol. 53, pp. 1015-1031, 2014.

24. J. Xu and J. Li, Stochastic dynamic response and reliability assessment of controlled structures with fractional derivative model of viscoelastic dampers, Mechanical Systems and Signal Processing, vol. 72-73, pp. 865-896, 2016.

25. M. Zayernouri and G.E. Karniadakis, Fractional spectral collocation methods for linear and nonlinear variable order FPDEs, Journal of Computational Physics, vol. 293, pp. 312-338, 2015.

26. J. Zhong and L. Li, Fractional-order system identification and proportional-derivative control of a solid-core magnetic bearing, ISA Transactions, vol. 53, pp. 1232-1242, 2014.

27. P. Zhuang, F. Liu, V. Anh and I. Turner, Numerical Methods for the Variable-Order Fractional Advection-Diffusion Equation with a Nonlinear Source Term, SIAM Journal on Numerical Analysis, vol. 47, pp. 1760-1781, 2009.

28. C. Zopf, S.E. Hoque and M. Kaliske, Comparison of approaches to model viscoelasticity based on fractional time derivatives, Computational Materials Science, vol. 98, pp. 287-296, 2015. 